\title{
Light vectors coupled to bosonic currents
}

\author{
Jeff A. Dror, ${ }^{1,2, *}$ Robert Lasenby, ${ }^{3, \uparrow}$ and Maxim Pospelov ${ }^{4,5, \$}$ \\ ${ }^{1}$ Department of Physics, University of California, Berkeley, California 94720, USA \\ ${ }^{2}$ Ernest Orlando Lawrence Berkeley National Laboratory, \\ University of California, Berkeley, California 94720, USA \\ ${ }^{3}$ Stanford Institute for Theoretical Physics, Stanford University, Stanford, California 94305, USA \\ ${ }^{4}$ Perimeter Institute for Theoretical Physics, 31 Caroline Street N, Waterloo, Ontario N2L 2Y5, Canada \\ ${ }^{5}$ Department of Physics and Astronomy, University of Victoria, \\ Victoria, British Columbia V8P 5C2, Canada
}

(Received 12 December 2018; published 12 March 2019)

\begin{abstract}
New spin-1 particles with masses below the weak scale are present in many theories of beyond Standard Model (SM) physics. In this work, we extend previous analyses by systematically considering the couplings of such a vector to the bosonic sector of the SM, focusing on models that lead to mass mixing with the $Z$ boson. These couplings generically lead to enhanced emission of the vector's longitudinal mode, both in Higgs decays and in flavor changing meson decays. We present bounds in the $\mathrm{SM}+X$ effective theory and investigate their model dependence. For the case of Higgs decays, we point out that tree-level vector emission is, depending on the model, not always enhanced, affecting the constraints. For meson decays, which are the dominant constraints at small vector masses, we find that while $B$ decay constraints can be weakened by fine-tuning UV parameters, it is generically difficult to suppress the stringent constraints from kaon decays.
\end{abstract}

DOI: $10.1103 /$ PhysRevD.99.055016

\section{INTRODUCTION}

Light new states, with masses below the weak scale, arise in many theories of physics beyond the Standard Model (SM). These may have gone undetected if their couplings to the SM are sufficiently weak. If such particles are not associated with many other new states at the same mass scale, then they should generically have spin $\leq 1$; here, we will concentrate on new light vector bosons, which have been extensively studied as mediators to a dark sector [1-3], and as explanations for experimental anomalies [4-9].

The lowest-dimensional interaction between a new vector and SM states is the dimension-4 coupling to a SM current, $X_{\mu} J_{\mathrm{SM}}^{\mu}$. Unless $J_{\mathrm{SM}}$ is conserved, including quantum effects, the $\mathrm{SM}+X$ effective theory is nonrenormalizable. This manifests as (energy $/ m_{X}$ )-enhanced amplitudes involving the longitudinal mode of $X$, for processes with $\partial_{\mu} J^{\mu} \neq 0$. Such processes can be the

\footnotetext{
*jdror@lbl.gov

rlasenby@stanford.edu

*mpospelov@perimeterinstitute.ca
}

Published by the American Physical Society under the terms of the Creative Commons Attribution 4.0 International license. Further distribution of this work must maintain attribution to the author(s) and the published article's title, journal citation, and DOI. Funded by SCOAP. dominant $X$ production mechanism at high-energy colliders, mostly importantly through emission in flavor changing neutral current (FCNC) $B$ and $K$ decays.

In previous works $[10,11]$, we systematically considered the phenomenological consequences of general couplings to SM fermions, $X_{\mu} J_{\mathrm{SM}, f}^{\mu}$. The only fully conserved currents in the SM are the electromagnetic (EM) current, and (if neutrinos are Dirac) the $B-L$ current; any other coupling will give rise to enhanced longitudinal emission in some circumstances (though if nonconservation is only through neutrino masses, e.g., for $L_{\mu}-L_{\tau}$, this will be very suppressed). Various models have been considered in the literature; light vectors having a chiral anomaly with the SM [10-12] (see also [13,14] for vectors above the weak scale), vectors with axial couplings [5,11,15-18], and ones with weak-isospin violation $[11,18]$.

Here, we extend our previous analyses by considering light vectors with couplings to bosonic SM fields. In particular, an $X W^{+} W^{-}$coupling will contribute to emission in FCNC penguin amplitudes, leading to similar phenomenology as for couplings to fermions ${ }^{1}$ [11]. New phenomenology can also arise from a coupling to the SM Higgs, leading to exotic Higgs decays. Such effects have been

\footnotetext{
${ }^{1}$ We can write the $W^{+} W^{-}$current as a sum of the EM current and a fermionic current, showing that longitudinal emission is the same as for the fermionic current.
} 
considered in a number of previous papers [19-21]; in this work, we consider the possibilities more systematically, investigating how signatures vary across different models.

For calculational simplicity, we will mostly work with UV completions that do not introduce extra electroweak symmetry breaking (EWSB). This means that, in the $\mathrm{SM}+X$ effective field theory (EFT), $X$ must couple to a SM-gauge-invariant current (or via Wess-Zumino terms, which are determined by the SM fermion couplings of $X$ $[10,11])$. The lowest-dimension couplings with nontrivial effects are

$$
J_{\mathrm{SM}, \mathrm{b}}^{\mu}=\partial_{\nu} B^{\mu \nu} ; \quad \partial^{\mu}\left(H^{\dagger} H\right) ; \quad H^{\dagger} i \stackrel{\leftrightarrow}{D} \mu, \quad H^{\dagger} i \stackrel{\leftrightarrow^{\mu}}{X} H
$$

where $D^{\mu}$ corresponds to the SM covariant derivative and $D_{X}^{\mu}$ includes a $\mathrm{U}(1)_{X}$ contribution, $D_{X}^{\mu} \equiv D^{\mu}+i g_{X} X^{\mu} / 2$. The first current in (1) is the familiar "kinetic mixing" operator [22], and upon the use of equations of motion for $B_{\mu}$ can be replaced with the hypercharge current $J_{Y}^{\mu}$. The $X_{\mu} \partial^{\mu}\left(H^{\dagger} H\right)$ coupling is equivalent, on integration by parts, to $-\left(\partial^{\mu} X_{\mu}\right) H^{\dagger} H$, and replacing $\partial^{\mu} X_{\mu}$ via the equations of motion gives a higher-dimensional operator.

The $H^{\dagger} D_{(X)} H$ currents are of more interest [the subscript $(X)$ is used to denote either the $\mathrm{SM}$ or $\left.\mathrm{SM}+\mathrm{U}(1)_{X}\right]$. In the unitary gauge, they lead to a mass mixing of $X$ with the $Z$ boson,

$$
\mathcal{L} \supset g_{X} X_{\mu}\left(H^{\dagger} i \stackrel{\leftrightarrow}{D}_{(X)}^{\mu} H\right) \rightarrow \varepsilon_{Z} m_{Z}^{2} X_{\mu} Z^{\mu}
$$

Transforming to the mass basis, this results in the light vector state gaining a coupling to the SM neutral current. In particular, this gives a coupling to $W^{+} W^{-}$, resulting in FCNC decays, and a coupling to the Higgs, resulting in exotic Higgs decays. Consequently, the mass mixing coupling provides an excellent prototype for the phenomenology of enhanced longitudinal emission. This coupling has also been the subject of several phenomenological studies (see, e.g., [19-21,23-33]), including motivations for new parity-violation experiments $[21,34,35]$.

Previous studies of $X-Z$ mixing have generally assumed that it leads to $1 / m_{X}$-enhanced amplitudes for $h \rightarrow Z X$ at tree level [19-21]. This is true if $\varepsilon_{Z} m_{Z}^{2} X_{\mu} Z^{\mu}$ is the only operator in the EFT, but not if it arises from the SM-gauge-invariant operators above. If we were to ignore the SM Yukawa couplings, then $H^{\dagger} D_{(X)} H$ would be conserved within the $\mathrm{SM}^{2}$ Consequently, the tree-level $h \rightarrow X Z$ amplitude is suppressed at small $m_{X}$. To obtain significant $1 / m_{X}$-enhanced amplitudes, we need to consider processes involving heavy fermions. Examples

\footnotetext{
${ }^{2}$ This is true for both currents for single $X$ emission, though $H^{\dagger} D H$ is not conserved in processes involving multiple $X$ 's, with consequences, e.g., in $h \rightarrow X X$.
}

include FCNC penguin amplitudes, and Higgs decays through a top loop, which we will study in detail.

The $h \rightarrow Z X$ amplitude is finite at one loop, so the rate for this processes is predicted in the EFT, up to corrections suppressed by the scale of heavier states. However, FCNC penguin amplitudes (and $h \rightarrow X X$ ) are divergent at one loop. Hence, in the limit that the scale of new states $\Lambda$ is significantly higher than the EW scale, the dominant contribution to the amplitude will scale as $\log \left(\Lambda^{2} / m_{\mathrm{EW}}^{2}\right)$. For SM-gauge-invariant $X$ couplings, the coefficient of this term is determined within the EFT. There will also be threshold corrections from the high-scale physics, which are not suppressed by inverse powers of $\Lambda$. Both of these effects mean that, without knowledge of the UV physics, only a generic prediction can be made for FCNC amplitudes.

If $\Lambda$ is not too high, then the log-enhanced term may be numerically comparable to other contributions. In particular, one might worry that there could be cancellations between this piece and other contributions, significantly altering the rates for meson decays. In this work, we illustrate the robustness of FCNC constraints within a two Higgs doublet model (2HDM), though we expect our conclusions to hold more generally. While $B \rightarrow K X$ rates can be suppressed by tuning the 2HDM parameters (in particular, the mass of the charged Higgs), $K^{ \pm} \rightarrow \pi^{ \pm} X$ amplitudes can only be suppressed by a factor of a few, unless the model has additional flavor structure.

The $1 / m_{X}$-enhanced amplitudes under discussion arise from the nonrenormalizability of the $\mathrm{SM}+X$ EFT, which must have a cutoff at (or below) a scale parametrically set by $m_{X} / g_{X}$. Standard UV completions, such as the 2HDM examples given later, result in higher-dimensional EFT operators involving $X$ being suppressed by $g_{X}$, as well as by inverse powers of the cutoff scale. Hence, the effects of dimension $>4$ operators are less important.

\section{FURTHER COMMENTS ON BOSONIC CURRENT PORTAL}

The $H^{\dagger} D_{(X)} H$ couplings have the following unitary gauge decomposition:

$$
\begin{gathered}
g_{X} X_{\mu} H^{\dagger} i \stackrel{\leftrightarrow^{\mu}}{D} H=m_{Z}^{2}\left(1+\frac{h}{v}\right)^{2} \varepsilon_{Z} X_{\mu} Z^{\mu}, \\
g_{X} X_{\mu} H^{\dagger} i \stackrel{\leftrightarrow}{D}_{X}^{\mu} H=m_{Z}^{2}\left(1+\frac{h}{v}\right)^{2}\left(\varepsilon_{Z} Z_{\mu}+\varepsilon_{Z}^{2} X_{\mu}\right) X^{\mu},
\end{gathered}
$$

where $v \simeq 246 \mathrm{GeV}$ is the electroweak vacuum expectation value (VEV), $h$ is the physical $(125 \mathrm{GeV}$ ) Higgs boson, and $\varepsilon_{Z} \equiv g_{X} v / m_{Z}$. The additional contribution in $D_{X}$ leads to terms proportional to $X_{\mu} X^{\mu}$, resulting in the current being conserved even in processes involving multiple $X$ 's (but still no SM Yukawa insertions). As mentioned above, this 
form is somewhat deceiving as one may expect it to lead to unsuppressed $h X_{\mu} X^{\mu}$ and $h X_{\mu} Z^{\mu}$ couplings. To see this is not the case we work in the mass basis where we parametrize the couplings as

$$
\begin{aligned}
\mathcal{L} \supset & C_{h X Z}\left(\frac{h}{v}+\frac{1}{2} \frac{h^{2}}{v^{2}}\right) X_{\mu} Z^{\mu} \\
+ & \frac{1}{2} C_{h X X}\left(\frac{h}{v}+\frac{1}{2} \frac{h^{2}}{v^{2}}\right) X_{\mu} X^{\mu},
\end{aligned}
$$

where the relation between the Higgs and Higgs-squared couplings is guaranteed by assuming that the direction of electroweak symmetry breaking is aligned with the Higgs. We find

$$
\begin{aligned}
& C_{h X Z}=-2 \varepsilon_{Z} m_{X}^{2}, \quad C_{h X X}=2 \varepsilon_{Z}^{2} m_{X}^{4} / m_{Z}^{2} \quad\left(H^{\dagger} D_{X} H\right) \\
& C_{h X Z}=-2 \varepsilon_{Z} m_{X}^{2}, \quad C_{h X X}=2 \varepsilon_{Z}^{2} m_{Z}^{2} \quad\left(H^{\dagger} D H\right) . \quad(6)
\end{aligned}
$$

We conclude that the $\mathcal{M}_{h \rightarrow Z X} \propto m_{X}^{-1}$ is not a generic consequence of $Z X$ mass mixing, as it cancels for the simplest SM-gauge-invariant realization. Notice, however, that if such mixing is realized as part of a more complicated current, e.g., $X_{\mu}\left(H^{\dagger} i \stackrel{\leftrightarrow}{D} \mu\right)\left(H^{\dagger} H\right) \supset Z_{\mu} X^{\mu}, m_{X}^{-1}$ behavior can appear even at tree level. This is because $\left(H^{\dagger} i \stackrel{\stackrel{D}{D}^{\mu}}{H}\right)\left(H^{\dagger} H\right)$ is not conserved in the purely bosonic sector. Similarly, if there are additional sources of EWSB, then this spoils the conservation of the $\mathrm{U}(1)_{X}$ current and can also lead to enhanced rates (as discussed below in the case of a $2 \mathrm{HDM}$ model).

While important for Higgs physics, these couplings with $h$ will not affect the one-loop results for the FCNC amplitudes. Therefore, the mass mixing $X_{\mu} Z^{\mu}$ operator and full SM-gauge-invariant couplings, $X \cdot H^{\dagger} D_{(X)} H$, at leading loop level will give identical answers for FCNCs (as will any model having the same $X W^{+} W^{-}$and $X$-quark vertices). Nevertheless, the SM-gauge-invariant theory is more convenient for calculations, as it allows these to be performed in arbitrary gauges. Keeping the Goldstone modes in the Lagrangian, in addition to the couplings in (3) or (4), we have

$$
\mathcal{L} \supset \frac{1}{2} g_{X} X^{\mu}\left(G^{+} i \partial_{\mu} G^{-}-g v G^{+} W_{\mu}^{-}+\ldots\right)+\text { H.c. }
$$

where the ellipses denote neutral component terms which will not be relevant for us here.

It is instructive to discuss possible UV completions of (3) and (4). If the breaking of $\mathrm{U}(1)_{X}$ occurs at a high energy scale $\Lambda$, then the vector portal under discussion is itself an effective operator,

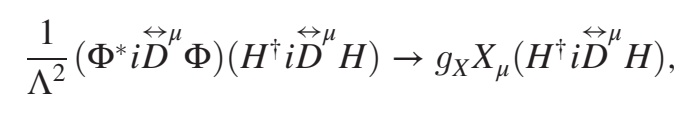

where $\Phi$ is the "dark" Higgs of $\mathrm{U}(1)_{X}$, and $D_{\mu}$ between $\Phi^{*}$ and $\Phi$ is the covariant derivative with respect to $\mathrm{U}(1)_{X}$, $D^{\mu}=\partial^{\mu}+i g^{\prime} X^{\mu}$, so that the coupling $X$ used previously relates to the $\mathrm{U}(1)_{X}$ charge $g^{\prime}$ as $g_{X}=g^{\prime}|\langle\Phi\rangle|^{2} / \Lambda^{2}$. Such couplings of $X$ were discussed in e.g., [36]. While explicitly and separately SM and $\mathrm{U}(1)_{X}$ invariant, operator (8) is of dimension 6 and needs further UV completion. A straightforward UV completion can be built using sets of heavy vectorlike fermions: $N_{\mathrm{SM}}$ (charged only under $\mathrm{SM}$ ), $N_{X}$ [charged only under $\mathrm{U}(1)_{X}$ ], and $N$ (singlet under all gauge symmetries). The charges are assigned in such a way that $\Phi N N_{X}$ and $H N N_{\mathrm{SM}}$ Yukawa interactions are allowed. This way, one fermionic loop of the $N-N_{X}-$ $N_{\text {SM }}$ mixed propagator creates (8), and the effective energy scale that would resolve this interaction is linked to the fermion masses.

A UV completion of (4) can be accomplished with a 2HDM model as considered in [24]. This introduces two $S U(2)_{L}$ doublets $H_{1}$ and $H_{2}$; the first is "SM-like," coupling to SM fermions $\left(\psi_{i}\right)$, while the second has no fermion couplings, but couples to $X$. Schematically,

$$
\mathcal{L} \supset D_{\mu} H_{1}^{\dagger} D^{\mu} H_{1}+y_{i j} H_{1} \psi_{i} \psi_{j}+D_{X}^{\mu} H_{2}^{\dagger} D_{X, \mu} H_{2},
$$

where the covariant derivative acting on $\mathrm{H}_{2}$ contains a $\mathrm{U}(1)_{X}$ contribution. To avoid introducing extra EWSB into the $\mathrm{SM}+X$ EFT requires the light scalar Higgs to be aligned with the direction of EWSB. In the notation of [37], this is the "decoupling limit" $\cos (\alpha-\beta)=0$, where $\alpha$ is the angle between the neutral components of $H_{2}, H_{1}$ and the scalar mass states, and $\beta$ is the angle between $H_{1}, H_{2}$ and the direction of EWSB. Then, writing $\mathrm{H}_{2}=s_{\beta} H+$ $c_{\beta} \tilde{H}$, where $H$ is the SM Higgs doublet and $\tilde{H}$ consists of heavier states that do not obtain a VEV, we can integrate out $\tilde{H}$ to obtain an effective coupling of the form of (4),

$$
\left(D_{X, \mu} H_{2}\right)^{\dagger}\left(D_{X}^{\mu} H_{2}\right) \supset \underbrace{-\frac{g_{X}}{g / 2 c_{W}} s_{\beta}^{2}}_{\varepsilon_{Z}} X_{\mu} H^{\dagger} i \stackrel{\leftrightarrow}{D}_{X}^{\mu} H .
$$

The mass of $X$ in this model receives two independent contributions: one from the VEV of $H$, and another from a Stueckelberg mass term in the Lagrangian. In more general 2HDM models, where $h$ is not aligned with the direction of EWSB, we obtain couplings beyond (3) and (4), allowing enhanced Higgs decay rates at tree level [19-21].

Lastly, one might wonder if it is possible to remove the dangerous $m_{X}^{-1}$-enhanced terms by charging additional fermions under $\mathrm{U}(1)_{X}$, resulting in a conserved current. The simplest way of doing it is suggested by the conservation of the hypercharge current, that can be split into bosonic and fermionic parts: 


$$
0=\partial_{\mu} J_{Y}^{\mu}=\partial_{\mu}\left(H^{\dagger} i \stackrel{\leftrightarrow}{D}{ }^{\mu} H+\sum_{i \in \mathrm{SM}} Y_{i} \bar{\psi}_{i} \gamma^{\mu} \psi_{i}\right)
$$

Thus, if the bosonic Higgs current is completed by the addition of the fermionic hypercharge current, one would remove $m_{X}^{-1}$-enhanced terms. This would correspond to the "usual" case of the kinetic mixing/hypercharge portal.

\section{FERMIONIC PROCESSES}

SM fermion masses result in nonconservation of the axial part of the neutral current, so the enhancement of longitudinal $X$ emission will occur the most in processes involving heavy fermions. For example, $X$-emission in the top-quark production and decay, $g g(q \bar{q}) \rightarrow t \bar{t} X$ or $t \rightarrow W b X$, will occur with the rate proportional to $\varepsilon_{Z}^{2} m_{t}^{2} / m_{X}^{2}$ [25,26]. Consequently, should this parameter be large, both the top-quark production cross section and its decay width will be affected. Given $O(5 \%-10 \%)$ accuracy in measuring the inclusive rates, setting very strong bounds on $\varepsilon_{Z}$ does not seem realistic. A somewhat better sensitivity could likely be achieved employing exclusive searches of light $X$ final states.

The most important constraints originating from the $X$-exchange are from $\nu_{e}-e$ scattering and atomic parity violation (APV). Neutrino scattering and APV are insensitive to the longitudinal mode of $X$, and at very small $m_{X}$ the constraints are $m_{X}$ independent. The most powerful limits from $\nu_{e}-e$ scattering are from a combination of the TEXONO [38-41], LSND [42], BOREXINO [43], GEMMA [44], and CHARM II [45] experiments. The authors of Ref. [46] summarized the limits for a $B-L$ vector and we expect similar limits to apply for a mass mixed vector with the identification, $g_{B-L} \leftrightarrow e \varepsilon_{Z}$. Since these are not constraining in any part of our parameter space we include these here using this rough approximation.

Researchers performing atomic parity-violation and electron parity-violation scattering experiments have long considered a mass-mixed vector as a prime target for their analyses $[47,48]$. We directly implement their limits here as described in $[49,50]$.

\section{HIGGS DECAYS}

The simplest processes involving bosonic couplings of $X$ are the exotic Higgs decays $h \rightarrow Z X$ and $h \rightarrow X X$, possible for $m_{X}<m_{h}-m_{Z}$ and $m_{X}<m_{h} / 2$ respectively. As per the discussion in previous sections, if the UV completion does not introduce extra EWSB, then the $h \rightarrow Z X$ rate is not enhanced as $m_{X} \rightarrow 0$, at least at tree level. For the $H^{\dagger} i D_{X}^{\mu} H$ coupling, the $h \rightarrow X X$ rate is likewise unenhanced at tree level. However, as we calculate in this section, loop-level effects from heavy fermions restore the small- $m_{X}$ enhancement.

At one loop the effective coupling is given by the diagrams shown in Fig. 1. Note that in principle one
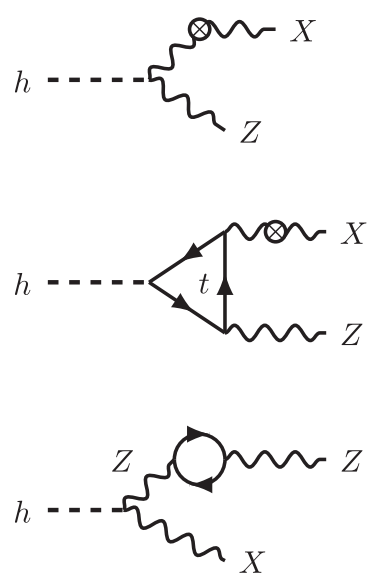
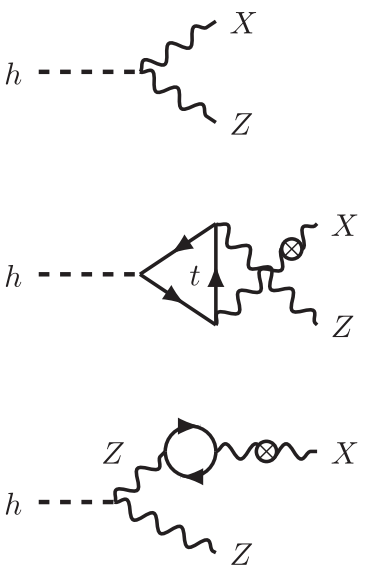

FIG. 1. Leading contributions to $h \rightarrow Z X$ decay at one-loop, where the " $\otimes$ " denotes a mass mixing. At tree level there is a cancellation between the mixing term and the direct $h Z X$ coupling. Similar diagrams are present for $h \rightarrow X X$ decays.

should also include mass and field renormalization corrections on the Higgs; however these corrections all lead to corrections suppressed by extra powers of $m_{X}$ and we drop them here. The effective operators are straightforward to compute with only the axial coupling contributions leading to operators of the form in (5).

After the inclusion of counterterms for the masses and the mixing parameter the divergence in the $h X_{\mu} Z^{\mu}$ is canceled, leaving behind a finite piece given by

$$
C_{h Z X}^{\text {loop }}=\varepsilon_{Z} \frac{N_{c} g_{Z}^{2} m_{t}^{2}}{(4 \pi)^{2}}
$$

where $g_{Z} \equiv e / s_{W} c_{W}$. These should be added with the treelevel couplings in (6) in either model to get the total couplings. Note that this expression has an opposite sign to the tree-level contribution and hence will interfere destructively when the two are comparable (at larger $m_{X}$ ).

Contrary to $h Z_{\mu} X^{\mu}$, the $h X_{\mu} X^{\mu}$ effective vertex is logdivergent and hence dependent on the UV completion. The effective vertex takes the form

$$
C_{h X X}^{\text {loop }}=\varepsilon_{Z}^{2} \frac{N_{c} g_{Z}^{2} m_{t}^{2}}{(4 \pi)^{2}} \log \frac{\Lambda^{2}}{m_{t}^{2}} .
$$

These give the decay rates

$\Gamma_{h \rightarrow Z X}=\frac{\left|C_{h Z X}\right|^{2} m_{h}}{16 \pi v^{2} m_{X}^{2}}\left[2 w+\frac{(1-w-z)^{2}}{4 z}\right] \sqrt{\lambda(1, w, z)}$,

$\Gamma_{h \rightarrow 2 X}=\frac{\left|C_{h X X}\right|^{2} m_{h}^{3}}{128 \pi v^{2} m_{X}^{4}}\left[1-4 w+12 w^{2}\right] \sqrt{\lambda(1, w, w)}$,

where $\quad \lambda(1, w, z) \equiv 1+w^{2}+z^{2}-2 z w-2 w-2 z \quad$ and $w \equiv m_{X}^{2} / m_{h}^{2}, z \equiv m_{Z}^{2} / m_{h}^{2}$. Note that these are of the same form as those computed at tree level [using Eq. (6)] but 
involve $C_{i}$ which are not suppressed by powers of $m_{X}$. As promised, the (loop-level) decay rates are always enhanced and scale as $m_{X}^{-2}$ and $m_{X}^{-4}$ respectively.

Such production of $X$ has been searched for by ATLAS [51,52]. The $h \rightarrow Z X$ only applies for a limited range of masses, $m_{h}-m_{Z}>m_{X}>15 \mathrm{GeV}$, due to the large $h \rightarrow Z Z^{*}$ background leading to a requirement of reconstructing the invariant mass of the decay products of $X$. On the other hand, $h \rightarrow X X$ applies in a broader mass range; $\mathrm{GeV} \lesssim m_{X}<m_{h} / 2$ though involves more powers of $\varepsilon_{Z}$, leading to slower improvement with luminosity. We recast the limits here in Fig. 3 with the solid lines corresponding to couplings to the $H^{\dagger} D_{X} H$ current and the dotted ones to couplings to $H^{\dagger} \mathrm{DH}$.

Interestingly we find that rare Higgs decays are already a competitive constraint in the high mass range. As expected the constraints are stronger for the $H^{\dagger} D H$ current, already surpassing atomic parity violation. Further we note that limits for $h \rightarrow Z X$ drop at lower masses due to a cancellation between the loop- and tree-level contributions. If experimental thresholds could be lowered then such constraints would also be enhanced at smaller $m_{X}$. Lastly we note that the solid line Higgs decay constraints also apply on the 2HDM of [19] in the alignment limit, with the new loop induced constraints becoming most important at lower masses. With more data we expect future searches for rare Higgs decays will be the most constraining for heavier vectors also coupled to the more evasive $H^{\dagger} D_{X} H$ current.

\section{FCNC AMPLITUDES WITH LONGITUDINAL $X$ EMISSION}

In this section we evaluate the amplitudes for FCNC decays via longitudinal $X$ emission, using the $\mathrm{SM}+X$ EFT. The SM flavor changing meson decay amplitudes are suppressed by the weak scale (schematically denoted $m_{\mathrm{EW}}$ ), whereas on-shell $X$ amplitudes may only be suppressed by the $X$ coupling. This can lead to FCNC decays via an on-shell longitudinal $X$ enhanced by $\left(m_{\mathrm{EW}} / m_{X}\right)^{2}$ relative to their SM counterparts, partially making up for the $g_{X}^{2}$ suppression. This effect, combined with the large statistics and high precision achieved in experimental studies of $K$ and $B$ decays (as opposed to e.g., Higgs boson decays), makes them a powerful tool in constraining $\left\{m_{X}, g_{X}\right\}$ parameter space.

Evaluating the down-type FCNC amplitude, $d_{i} d_{j} X$, in the $\mathrm{SM}+X$ EFT, involves the sum of diagrams in Fig. 2 (as a consequence of not including additional sources of EWSB there is no $X W^{+} W^{-}$vertex in this basis). Diagrams (a) and (b) involve the $X G^{+} G^{-}$and $X G^{+} W^{-}$couplings, while (c)-(i) come from the $X_{\mu} Z^{\mu}$ mixing. In an $R(\xi)$ gauge, the amplitude from (c)-(i) is, at leading order in external momenta, simply $\varepsilon_{Z}$ times the SM Z-penguin result [53], which is finite. However, the charged Goldstone coupling in (a) results in an uncanceled logarithmic
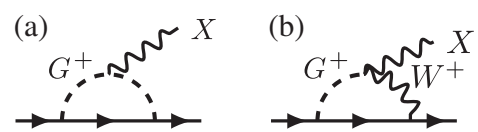

(c)

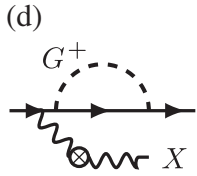

(e)

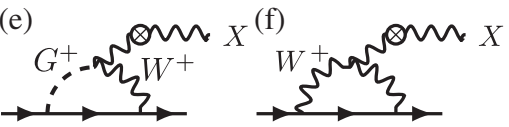

(g)

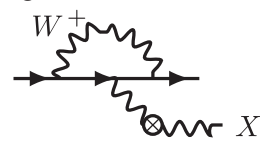

(h)
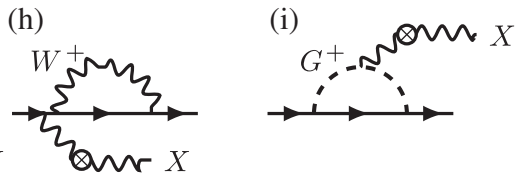

FIG. 2. (a)-(i) represents the diagrams used to compute the $b s X$ amplitude from the SM $+X$ EFT for $X$ coupled to either the $H^{\dagger} D H$ or $H^{\dagger} D_{X} H$ current. The $\otimes$ symbol denotes a mass mixing between the $X$ and $Z$. Diagrams (b) and (e) include swapping the $G^{+}$and $W^{+}$lines while (d) and (h) include emitting the $Z$ from the outgoing fermion lines.

divergence. Writing the total amplitude in terms of a low-energy effective interaction, we have

$$
\begin{gathered}
\mathcal{L} \supset g_{d_{i} d_{j} X} \bar{d}_{i} \gamma_{\mu} P_{L} d_{j} X^{\mu}, \\
g_{d_{i} d_{j} X} \simeq \frac{g^{3}}{2 c_{W}} \frac{\varepsilon_{Z}}{16 \pi^{2}} \sum_{q \in\{u, c, t\}} V_{q d_{i}} V_{q d_{j}}^{*} f\left(m_{q}^{2} / m_{W}^{2}\right),
\end{gathered}
$$

where $f$ depends on the UV parameters. Assuming a wide scale separation, and the dominance of the logarithmic contribution, in the EFT one finds

$$
f(x)=-\frac{x}{4} \log \frac{\Lambda^{2}}{m_{W}^{2}}+c_{\mathrm{th}},
$$

where $c_{\text {th }}$, associated with threshold corrections, is subleading to the logarithmic part in the limit where the high scale $\Lambda \gg m_{W}$. As usual, these threshold corrections come from both the IR scales (associated here with the weak scale) and the UV scale. Strictly speaking, it is only within a specific UV completion that one could fully identify the scale $\Lambda$ and define $c_{\text {th }}$. In particular, while the coefficient of the log-enhanced term in the EFT calculation is gauge independent, the other parts of the amplitude are not, illustrating that they depend on the UV completion. If additional states are at a sufficiently high scale, then we would expect the log-enhanced term to dominate, allowing concrete constraints to be set. Conversely, for smaller $\Lambda$, threshold corrections can be significant, opening the possibility of canceling it against the log-divergent piece. We discuss this more quantitatively in an explicit UV completion in Sec. VI.

In unitary gauge, only the $Z$-mixing diagrams contribute, and the amplitude is just given by $\varepsilon_{Z}$ multiplied by the SM 


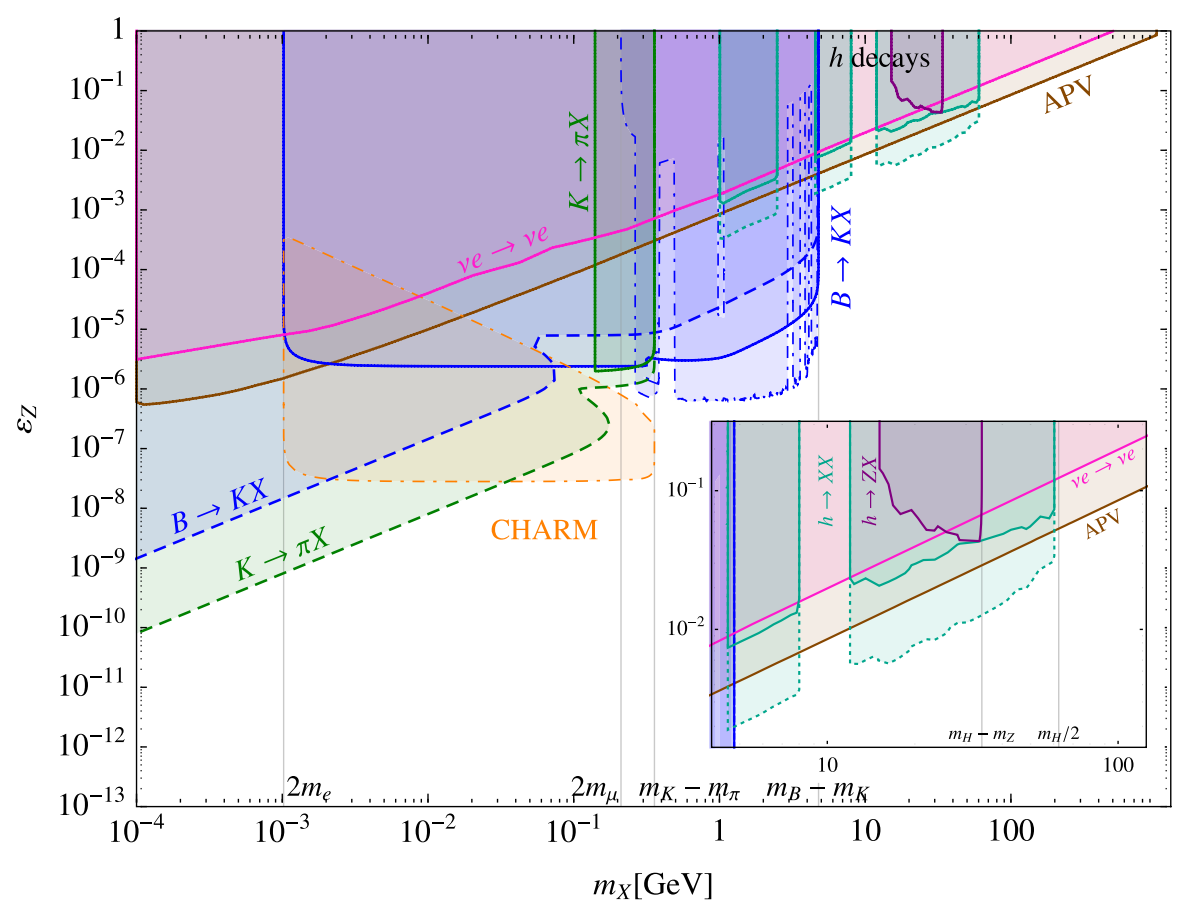

FIG. 3. Constraints on a vector $X$ with a "mass-mixing" coupling $\varepsilon_{Z} m_{Z}^{2} Z_{\mu} X^{\mu}$ to the SM $Z$ boson. The FCNC rates from such a coupling are UV dependent - the degree to which this can affect constraints is discussed in the text. We use the limits on $B$ decays from [55-57], from [58,59] for $K$ decays ([60] for the SM prediction for the $K \rightarrow \pi \bar{\nu} \nu$ decay), from [61] for the CHARM beam dump, from [46] for $\nu-e$ scattering, and finally from [49,50] for computing the constraints on atomic parity violation. Solid lines denote constraints arising from visible searches, dashed from invisible searches, and dot-dashed for displaced searches. The Higgs decay constraints arise from direct searches for $h \rightarrow X X$ and $h \rightarrow Z X$ with the solid color corresponding to a vector coupled to $H^{\dagger} D_{X} H$ and the dotted to a coupling to $H^{\dagger} \mathrm{DH}[52]$.

Z-penguin. This may be slightly confusing, since amplitudes for physical processes in the SM must be finite. Indeed, for the loop-induced FCNC decay of an on-shell $Z$-boson, $Z \rightarrow d_{i} \bar{d}_{j}$, such divergence is absent. The explanation is that the $Z$ mass in the SM is related to its couplings, in a way that makes the divergences in the externalmomenta-dependent terms in the FCNC amplitude (which we neglected above) exactly cancel the momentumindependent divergences [54]. For SM FCNC amplitudes involving off-shell $Z$ exchange, such as $d_{i} \rightarrow d_{j} l \bar{l}$, divergences in the effective $d_{i}-d_{j}-Z$ vertex cancel against the divergences from $W$ box diagrams [53,54]. However, $m_{X}$ and $g_{X}$ are independent parameters, so this cancellation will not occur for FCNC decays via an on-shell $X$. Consequently, computing in unitary gauge, where diagram (a) is absent, also gives rise to divergences in the $\mathrm{SM}+X$ EFT, resulting in the same $\log \Lambda^{2} / m_{W}^{2}$ contribution as the $R(\xi)$ gauge calculation.

While the top-quark loop will in general dominate, it is also useful to determine the EFT answer for the light quark contributions. If all of the up-type quarks had the same mass, then Cabibbo-Kobayashi-Maskawa unitarity would mean that the sum in Eq. (17) cancels-at least, that the EFTcalculable parts do. A UV completion with additional flavor structure could lead to the $c_{\text {th }}$ contributions varying between different up-type quarks. However, for a UV completion without such structure, without loss of generality we can take $f(x) \rightarrow 0$ as $x \rightarrow 0$, since all up-type quarks being massless would lead to zero total amplitude. Within the EFT, $f(x) \propto x \log x$ for small $x$. This means that even for an $s-d-X$ vertex, the internal up and charm contributions are always subdominant to the internal top one (unless special cancellations occur), despite the corresponding CabibboKobayashi-Maskawa elements being larger.

To summarize, FCNC amplitudes in the $\mathrm{SM}+X$ EFT run logarithmically, so are dependent on the UV physics. However, their generic scale is set by the $X$ couplings in the EFT. The same structure applies for $X$ couplings to SM fermions, as analyzed in [11]. For sufficiently light $X$, all of these lead to FCNC meson decays with rates enhanced by $\sim m_{\mathrm{EW}}^{2} / m_{X}^{2}$ compared to competing SM processes (this is for down-type FCNCs - up-type FCNC rates are suppressed by at least additional powers of the charm mass over the weak scale). As discussed in [11], the corresponding $B$ and $K$ meson decays can provide strong constraints on nonrenormalizable $X$-SM couplings. Figure 3 illustrates these constraints for a "typical" UV model by taking $\Lambda \sim \mathrm{TeV}$ in Eq. (18), and including only the log-enhanced term. In the next section, we will see how this relates to the results in an example UV theory. The figure also shows 
other constraints arising from the SM couplings of $X$, including neutrino-electron scattering and parity-violation experiments, illustrating that when FCNC constraints apply, they are generically significantly stronger than other probes.

As mentioned in the Introduction, any model that results in an $X W^{+} W^{-}$vertex (and/or couplings to quarks) will generically contribute to FCNCs in a similar way. The $\varepsilon_{Z}$ model here is illustrative in that it results in UV-divergent and $m_{t}^{2} / m_{W}^{2}$-enhanced amplitudes (in contrast to e.g., Wess-Zumino couplings, which lead to finite FCNC amplitudes $[10,11])$. As in the case of couplings to SM fermions [11], this is the generic and strongest behavior.

\section{A. Beam-dump constraints}

In our previous work [10,11], we stated that $K \rightarrow \pi X_{L}$ decays would be the dominant $X$ production mechanism in proton beam-dump experiments such as CHARM [61], following the analysis in [62]. However, the details of this analysis are incorrect, since almost all of the kaons produced in such experiments will be stopped in the target before decaying. ${ }^{3}$

The consequences of kaon interactions in the target were investigated by the author of [63], who adopted the conservative procedure of estimating the number of kaons that decay before their first interaction. Considering $X$ production from only these kaon decays results in a significantly smaller yield than we used in [10,11]. However, it is still the case that $K \rightarrow \pi X_{L}$ decays are only suppressed by $m_{\mathrm{EW}}^{2} /\left(m_{X}^{2} / g_{X}^{2}\right)$ compared to SM channels. On the other hand, the production rate from " $\pi^{0}$-like" interactions, as estimated in [61,64], is suppressed by $\sim f_{\pi}^{2} /\left(m_{X}^{2} / g_{X}^{2}\right)$ compared to the SM $\pi^{0}$ production rate. Consequently, even taking the small kaon survival rate from [63], $X$ production from kaon decays still dominates over the $\pi^{0}$ estimate. In addition to $X$ produced from kaon decays, there is a subdominant component arising from the production of $B$ mesons, which decay into vectors with significantly larger boosts [65]. However, due to the smaller primary yield of $B$ mesons (using the estimate in [63]) $X$ production from kaons, when kinematically allowed, is typically larger. Our constraints from the CHARM beam-dump experiment (plotted in Figs. 3 and 5), which use the kaon and B efficiency estimates from [63], illustrate that beam dumps can still constrain new parameter space.

Neutrino beam experiments, in which pions and kaons are not stopped in the target, but instead focused into a collimated beam, result in a much larger kaon decay yield, and would consequently give more $X$ production. However, the detectors in such experiments are generally set up to detect neutrino scattering events, which would constitute a

\footnotetext{
${ }^{3}$ We thank Felix Kahlhoefer for bringing this issue to our attention.
}

background to the $X$ decays we want to search for. In contrast, the beam-dump mode run of CHARM [61] used a large, air-filled detector volume, yielding a basically background-free experiment (this difference is illustrated in [66]).

\section{2HDM EXAMPLE}

The $\mathrm{SM}+X$ EFT calculation in the previous section gives the "generic" scale of FCNC amplitudes. However, for UV completions in which new physics is not too far above the EW scale, it is possible that other contributions will be numerically comparable to the log-enhanced term. In particular, one might wonder about the robustness of limits for particular UV completions, as partial cancellations in the amplitude may weaken FCNC constraints. In this section, we will perform a full calculation of FCNC amplitudes (to leading order in external momenta) within a simple UV theory, illustrating these points.

As our reference model we use the 2HDM summarized in Sec. II. Calculating FCNC vertices in the full $2 \mathrm{HDM}$ theory, the UV divergences coming from EW penguin diagrams are canceled by diagrams involving charged Higgs exchange (Fig. 4). Computing these, we find that the total contribution to the FCNC amplitude is given by

$$
g_{d_{i} d_{j} X} \simeq-\frac{g_{X} g^{2} s_{\beta}^{2}}{16 \pi^{2}} \sum V_{q d_{i}} V_{q d_{j}}^{*} f\left(\frac{m_{q}^{2}}{m_{W}^{2}}, \frac{m_{H^{+}}^{2}}{m_{W}^{2}}\right),
$$

where

$$
\begin{aligned}
f(x, y) \equiv & \frac{x}{4}\left\{2+\frac{y}{y-x}-\frac{3}{x-1}+\frac{3(y-2 x+1)}{(y-1)(x-1)^{2}} \log x\right. \\
& \left.+\frac{y\left(y^{2}-7 y+6 x\right)}{(y-1)(y-x)^{2}} \log \frac{x}{y}\right\}
\end{aligned}
$$

and we use $x_{q} \equiv m_{q}^{2} / m_{W}^{2}$ and $y \equiv m_{H^{+}}^{2} / m_{W}^{2}$.

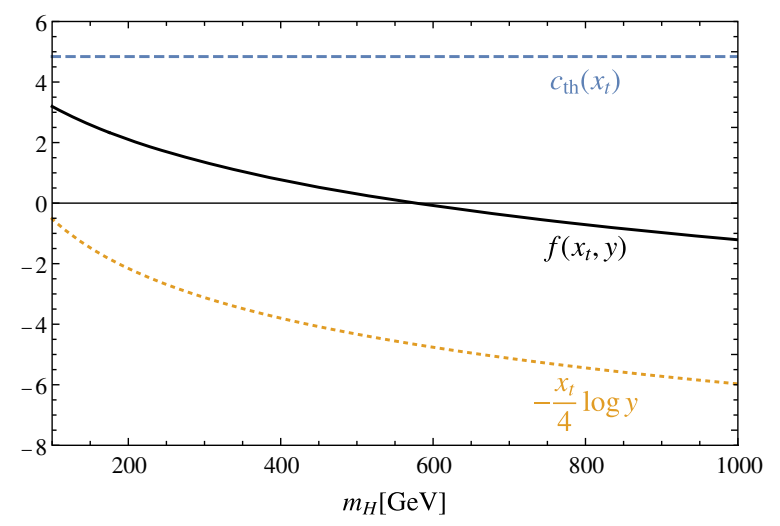

FIG. 4. Different contributions to the effective $d_{i} d_{j} X$ coupling from top loops as given in Eq. (20) (with $x_{t} \equiv m_{t}^{2} / m_{W}^{2}$ ). The dashed blue and dotted orange lines denote the threshold and largelogarithm pieces respectively while the black line denotes the full one-loop function. We see a cancellation between the threshold and running contributions around $m_{H} \simeq 575 \mathrm{GeV}$ beyond which the coupling is well approximated by the log-enhanced piece. 
For $y$ large, $f(x, y) \simeq-(x / 4) \log y$, in agreement with the EFT result in Eq. (17). Identifying the scale $\Lambda$ with $m_{H^{+}}$, we also find the threshold correction in the limit of large $\Lambda$,

$$
c_{\mathrm{th}}=\frac{x\left(3 x^{2}-9 x+6+\left(x^{2}-2 x+4\right) \log x\right)}{4(x-1)^{2}} .
$$

It is easy to see that for $x$ given by the top mass, the sign of $c_{\text {th }}$ is opposite to $-\frac{1}{4} x \log y$. For small enough $x$, we have $f \simeq x \log x$, again as expected from the EFT. Our results are in agreement with results in previous studies [24] which computed the amplitude by relating the theory to that of an axion with fermionic couplings [67]. As discussed in the previous section, the top-quark contribution is typically dominant, for both $K$ and $B$ decays.

The different threshold and running contributions are compared with the full expression in Fig. 4 showing a partial cancellation in the flavor changing rate at $m_{H^{+}} \simeq$ $575 \mathrm{GeV}$ for the top contribution. Note, however, that since there are additional contributions to the total FCNC decay amplitude that have a different complex phase to the topquark contribution, the total amplitude does not pass through zero as we change $m_{H^{+}}$. For kaon decays, the next most important contribution to the $s d X$ vertex is from the charm-quark contribution. The argument ${ }^{4}$ of $V_{c s} V_{c d}^{*} \simeq$ $-0.22+10^{-4} i$ is significantly smaller than that of $V_{t s} V_{t d}^{*} \simeq$ $10^{-4}(-3.3-1.4 i)$, and to a large extent this mitigates the small charm mass, $f\left(x_{c}, y\right) / f\left(x_{t}, y\right) \propto \mathcal{O}\left(m_{c}^{2} / m_{t}^{2}\right)$. Consequently, as illustrated in Fig. 5, the limits from $K^{ \pm} \rightarrow \pi^{ \pm} X$ decays can only be relaxed by a factor of a few by tuning $m_{H^{+}}$. On the other hand, for $B$ decays, the charm-quark Cabibbo-Kobayashi-Maskawa product $V_{c b} V_{c s}^{*} \simeq 0.04+10^{-6} i$ has a similar magnitude to the top-quark product, $V_{t b} V_{t s}^{*} \simeq-0.04+7 \times 10^{-4} i$, and the charm loop will not play an important role (the cancellation will instead mostly be lifted by external momentum corrections, which are suppressed by $\sim m_{b}^{2} / m_{W}^{2}$ compared to the top contribution).

Figure 5 illustrates how these cancellations affect the experimental constraints on the coupling of the new vector, for a representative value of $m_{X}=300 \mathrm{MeV}$ (where both $K$ and $B$ decay constraints apply). As noted above, for "generic" UV completions, the FCNC constraints are significantly stronger than other experimental probes. The figure shows that $B \rightarrow K X$ and $K_{L} \rightarrow \pi^{0} X$ constraints (the latter process is $C P$ violating, so only depends on the imaginary part of the amplitude, which generically goes through zero) can be significantly relaxed at the same value of $m_{H^{+}}$. However, the $K^{ \pm} \rightarrow \pi^{ \pm} X$ constraints are not significantly canceled around that point; overall, the limit

\footnotetext{
${ }^{4}$ We use the Particle Data Group parametrization of the Cabibbo-Kobayashi-Maskawa matrix [68].
}

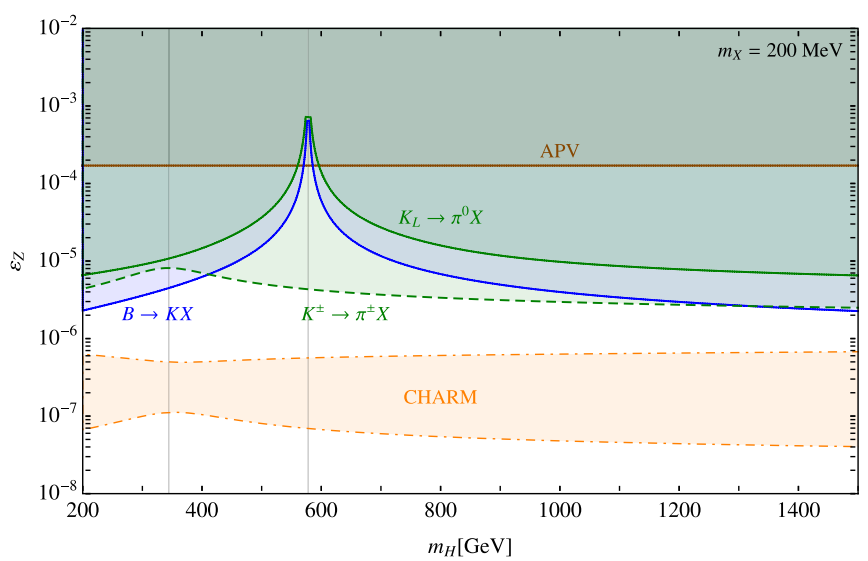

FIG. 5. Constraints on $\varepsilon_{Z}$ in the 2HDM model of Sec. VI, for a light vector mass of $m_{X}=300 \mathrm{MeV}$. As discussed in the text, varying the UV physics (here, the charged Higgs mass) changes the FCNC amplitudes involving $X$, and consequently changes FCNC meson decay rates. For $K^{+} \rightarrow \pi^{+} X$ decays, the impact is mild, whereas for $B \rightarrow K X$ and $K_{L} \rightarrow \pi^{0} X$ decays, there is a specific charged Higgs mass for which the rates are significantly reduced. We also include constraints from the CHARM beamdump experiment [61] which are dominated by production of both $K^{+}$and $K_{L}$. Also plotted are atomic parity-violation constraints $[49,50]$ on $\varepsilon_{Z}$, which do not depend on the UV completion-these are always weaker than $K^{+}$decay constraints, and can only compete with the other meson decays in a narrow $m_{H^{+}}$range.

on $g_{X}$ varies only by a factor $\sim 2$ across the entire range of $m_{H^{+}}$. Nevertheless, the reduction of the log contributions due to threshold corrections is clearly an important effect. While constraints in Fig. 3 are plotted using the simplified form $f\left(x_{t}, y\right)=-\left(x_{t} / 4\right) \times \log \left(\mathrm{TeV}^{2} / m_{W}^{2}\right)$, the same value of $f(x, y)$ in the UV complete version is achieved with $m_{H^{+}} \simeq 7.5 \mathrm{TeV}$.

While these calculations were performed in the context of a particular UV completion, the generic point that different components of the amplitude have different complex phases can be seen within the EFT. Consequently, if FCNCs in the UV completion are dominantly controlled by one parameter $\left(m_{H^{+}}\right.$in our $2 \mathrm{HDM}$ example), then varying this parameter will generically not result in the amplitude passing through zero. Thus, unless the UV completion has additional flavor structure that enables it to arrange such a cancellation, and/or multiple parameters which affect the different-phase components in different ways, FCNC amplitudes can generically only be canceled by a similar degree to that derived here.

Another point illustrated by this UV completion is that the scale of new physics has to be sufficiently low for the partial cancellations to occur. For the case of extended Higgs sectors, the current constraints on the mass of the new charged Higgs states range from $\sim 200-600 \mathrm{GeV}$ depending on the details of the Higgs sector (see [69-71] for CMS searches and [72-74] for corresponding ATLAS searches). These limits are statistics dominated and should 
improve with the high luminosity LHC run. In the considered UV completion, the most relaxed bounds on $g_{X}$ are for $m_{H^{+}}$of $575 \mathrm{GeV}$, which is within reach of the LHC.

\section{KINETIC MIXING AND FCNCS}

The only fully renormalizable coupling of a new light vector to the SM is via "kinetic mixing" with the hypercharge gauge boson,

$$
\mathcal{L} \supset \frac{1}{2} \varepsilon_{Y} X_{\mu \nu} B^{\mu \nu}
$$

(if neutrinos are Dirac, then a coupling to the $B-L$ current is also renormalizable). Defining electromagnetic and weak neutral currents of the SM as $\mathcal{L} \supset A_{\mu} J_{\mathrm{EM}}^{\mu}+Z_{\mu} J_{Z}^{\mu}$, to first order in the small mixing $\varepsilon_{Y}$, we can perform field redefinitions so that the new light spin-1 state has couplings

$$
\mathcal{L} \supset \varepsilon_{Y} X_{\mu}\left(J_{\mathrm{EM}}^{\mu} \cos \theta_{W}+\frac{m_{X}^{2}}{m_{Z}^{2}} J_{Z}^{\mu} \sin \theta_{W}\right),
$$

where $\theta_{W}$ is the weak mixing angle. While a coupling to the (nonconserved) neutral current leads to energy $/ m_{X^{-}}$ enhanced longitudinal amplitudes, the $m_{X}^{2} / m_{Z}^{2}$ suppression of this coupling means that such amplitudes are suppressed by $m_{X} E / m_{Z}^{2}$ overall, and hence vanish for $m_{X} \rightarrow 0$ as required by renormalizability.

Most treatments of "dark photons"- - new vectors with a coupling to the EM current in the low-energy (sub-EWscale) SM-do not consider the small neutral current coupling that would arise from hypercharge mixing. One reason is that, in nonrenormalizable $\mathrm{SM}+X$ models, these couplings are not necessarily linked (e.g., one could always add a kinetic mixing to the models in the previous section). Another is that, while the neutral current coupling does give some experimentally distinct signatures, the $m_{X}^{2} / m_{Z}^{2}$ suppression means that, for $m_{X} \ll m_{Z}$, these are generally less important than the EM coupling signatures.

This point is illustrated by FCNC meson decays, which, as discussed in previous sections, are generally the strongest probes of a light vector's neutral current coupling. For kaon decays, the $K^{+} \rightarrow \pi^{+} \gamma^{*}$ amplitude can be computed in chiral perturbation theory, using information on the $K \rightarrow 3 \pi$ amplitude as input [75]. This gives the FCNC amplitude from the EM current coupling of $X$ [6]. The amplitude from the neutral current couplings is given by the usual EW penguin contribution. Due to the $m_{X}^{2}$ suppression of the neutral current coupling in (23), both of these are $\propto m_{X}^{2}$. However, the penguin contribution is suppressed by $V_{t s} V_{t d}^{*}$, which has magnitude $\sim 4 \times 10^{-4}$, whereas the $\gamma^{*}$ amplitude is only suppressed by the Cabibbo angle. Hence, the neutral current contribution to these decays is subdominant to the dark photon contribution. The same is true for $K_{S} \rightarrow \pi X$ decays. For $K_{L} \rightarrow \pi^{0} X$ decays, which are $C P$ violating, the neutral current term dominates; however, the experimental limits on these decays are comparable to those for $K^{+} \rightarrow \pi^{+} X$, so the latter will generally be a better probe of a hypercharge-mixed vector. In the end, it turns out that the kaon decays do not put any additional restrictions on dark photons compared to standard "bump hunt" searches, with the exception of models with a large $X \rightarrow$ invisible channel, where the competing SM rate, $K^{+} \rightarrow \pi^{+} \nu \bar{\nu}$, has a very small branching.

$B \rightarrow K X$ constraints on the kinetic mixing are even less important than the kaon constraints. $B \rightarrow K \gamma$ decay occurs with the branching ratio of $\simeq 4 \times 10^{-5}$, and the corresponding decay to a dark photon will be smaller by a factor of $\left(\varepsilon_{Y} \cos \theta_{W}\right)^{2}$. Current experimental constraints require $\varepsilon_{Y} \lesssim 10^{-3}$ over the relevant mass range, which renders the $B$ decays well outside experimental capabilities. Contributions of neutral currents do not change this conclusion.

\section{CONCLUSIONS}

We have discussed some of the simplest ways of coupling new light vectors to the bosonic sector of the SM and their phenomenological consequences. In particular, we have focused on the lowest-dimensional, SMgauge-invariant currents to which a new vector can couple. Besides kinetic mixing, the only two nontrivial currents are $H^{\dagger} D H$ and $H^{\dagger} D_{X} H$. Coupling a light vector, $X_{\mu}$, to these currents leads to an effective $Z-X$ mass mixing, as well as couplings of $X$ to the scalar Higgs. The most observationally significant effects of these couplings come about through $m_{X}^{-1}$-enhanced longitudinal $X$ emission, analogously to couplings to nonconserved fermionic currents [11].

An important property of the $H^{\dagger} D_{(X)} H$ currents is that they are conserved in the purely bosonic sector of the SM for single- $X$ emissions. Hence, one should not expect an enhancement of the longitudinal $X$ emission at tree level. Nevertheless, this property is broken by quantum corrections and $m_{X}^{-1}$-behavior is present when fermions are included, as the Yukawa/mass terms break current conservation. An important place to look for the source of the constraints is the loop-induced processes which can profit from the high energies available in the virtual states. Loops of top quarks can induce important signals for both currents of interest and we highlight their potential to probe such vectors.

For sufficiently light vectors, flavor changing decays of the $K$ and $B$ mesons are extremely sensitive probes of bosonic couplings, imposing more stringent constraints than parity-violation or scattering experiments. Applying the EFT calculation to the $\mathrm{SM}+X$ theory, we compute the FCNC amplitudes, identifying the leading $\log \left(\Lambda / m_{W}\right)$ behavior. To explore the relation of the effective theory to its UV completions and the possibility of a cancellation 
eliminating these constraints, we study a two Higgs doublet UV completion in detail, with one of the Higgs fields charged under $\mathrm{U}(1)_{X}$. We find that the threshold corrections, as it is often the case, contribute with the opposite sign to the logarithmically enhanced terms. At $m_{H^{+}} \sim$ $575 \mathrm{GeV}$ there is nearly perfect cancellation of the $b s X$ amplitude. We argue, however, that such cancellation is not universally applicable to all enhanced $d_{i} d_{j} X$ amplitudes. Up to the caveat of an exotic case of UV completion with additional flavor structure, FCNC contributions with different complex phases cannot generically be simultaneously canceled, leading to the conclusion that $K^{+} \rightarrow \pi^{+} X$ amplitudes can only be suppressed by a factor of a few.

Another possibility raised in the literature [24] is that FCNCs could be canceled between nonrenormalizable and renormalizable (kinetic mixing) couplings of the same vector. However, such tuning is only relevant for $K^{ \pm} \rightarrow$ $\pi^{ \pm} X$ decays (and even then can only reduce the rate by a factor $\sim 10$ ); $B$-physics is far less affected by the kinetic mixing operators (and moreover $B \rightarrow K X$ and $B \rightarrow K^{*} X$ decays are affected differently).

While we performed FCNC calculations for particular vector coupling models, these conclusions apply more generally. Quark FCNC amplitudes depend on the $X W^{+} W^{-}$ coupling (and the quark couplings) of the new vector, so any models giving that coupling will lead to the similar decays. Other couplings of $X$ to SM bosons have smaller effects; a $X Z Z$ vertex only contributes to less important low-energy processes, while processes involving vertices of $X$ with three SM gauge bosons are suppressed by additional powers of the weak scale (or extra loop factors). There can also be dimension- 4 couplings involving more than one $X$, such as an $X X W^{+} W^{-}$vertex. However, if the $X$-SM couplings are all suppressed by some small parameter $g_{X}$, then in most UV completions, $X X$-SM vertices will be suppressed by $g_{X}^{2}$, limiting their capability to constrain these couplings.

For heavier vectors, exotic Higgs decays provide a relatively clean probe, as long as the vector decays to SM states. We studied the decays for both currents, finding that only for $H^{\dagger} D H$ can the tree-level decays have a longitudinal enhancement, observable in $h \rightarrow X X$ decays. Nevertheless, approximate current conservation is broken at one loop leading to enhanced $h \rightarrow Z X$ and $h \rightarrow X X$ decays for both currents. These conclusions rely on our assumption that the theory does not have additional sources of electroweak symmetry breaking other than the SM Higgs. If this is violated (e.g., by a generic two Higgs doublet model), then enhanced decays can occur already at tree level. Overall, we clarify how Higgs decay rates to $X$ can vary across different models, and we highlight their potential to probe vectors above the few-GeV meson threshold.

\section{ACKNOWLEDGMENTS}

Research at Perimeter Institute is supported by the Government of Canada through Industry Canada and by the Province of Ontario through the Ministry of Economic Development and Innovation. J. A. D. is supported in part by the DOE under Contract No. DE-AC02-05CH11231.
[1] C. Boehm and P. Fayet, Nucl. Phys. B683, 219 (2004).

[2] M. Pospelov, A. Ritz, and M. B. Voloshin, Phys. Lett. B 662, 53 (2008).

[3] N. Arkani-Hamed, D. P. Finkbeiner, T. R. Slatyer, and N. Weiner, Phys. Rev. D 79, 015014 (2009).

[4] S. N. Gninenko and N. V. Krasnikov, Phys. Lett. B 513, 119 (2001).

[5] Y. Kahn, M. Schmitt, and T. M. P. Tait, Phys. Rev. D 78, 115002 (2008).

[6] M. Pospelov, Phys. Rev. D 80, 095002 (2009).

[7] D. Tucker-Smith and I. Yavin, Phys. Rev. D 83, 101702 (2011).

[8] B. Batell, D. McKeen, and M. Pospelov, Phys. Rev. Lett. 107, 011803 (2011).

[9] J. L. Feng, B. Fornal, I. Galon, S. Gardner, J. Smolinsky, T. M. P. Tait, and P. Tanedo, Phys. Rev. D 95, 035017 (2017).

[10] J. A. Dror, R. Lasenby, and M. Pospelov, Phys. Rev. Lett. 119, 141803 (2017).

[11] J. A. Dror, R. Lasenby, and M. Pospelov, Phys. Rev. D 96, 075036 (2017).

[12] A. Ismail and A. Katz, J. High Energy Phys. 04 (2018) 122.
[13] A. Ismail, A. Katz, and D. Racco, J. High Energy Phys. 10 (2017) 165.

[14] P. J. Fox, I. Low, and Y. Zhang, J. High Energy Phys. 03 (2018) 074.

[15] P. Fayet, Phys. Rev. D 74, 054034 (2006).

[16] P. Fayet, Phys. Rev. D 75, 115017 (2007).

[17] V. Barger, C.-W. Chiang, W.-Y. Keung, and D. Marfatia, Phys. Rev. Lett. 108, 081802 (2012).

[18] S. G. Karshenboim, D. McKeen, and M. Pospelov, Phys. Rev. D 90, 073004 (2014); 90, 079905(A) (2014).

[19] H. Davoudiasl, H.-S. Lee, and W. J. Marciano, Phys. Rev. D 85, 115019 (2012).

[20] H. Davoudiasl, H.-S. Lee, I. Lewis, and W. J. Marciano, Phys. Rev. D 88, 015022 (2013).

[21] H. Davoudiasl, H.-S. Lee, and W. J. Marciano, Phys. Rev. D 92, 055005 (2015).

[22] B. Holdom, Phys. Lett. 166B, 196 (1986).

[23] H.-S. Lee and M. Sher, Phys. Rev. D 87, 115009 (2013).

[24] H. Davoudiasl, H.-S. Lee, and W. J. Marciano, Phys. Rev. D 89, 095006 (2014). 
[25] D. Kim, H.-S. Lee, and M. Park, J. High Energy Phys. 03 (2015) 134.

[26] K. Kong, H.-S. Lee, and M. Park, Phys. Rev. D 89, 074007 (2014).

[27] H. Davoudiasl, W. J. Marciano, R. Ramos, and M. Sher, Phys. Rev. D 89, 115008 (2014).

[28] F. Xu, J. High Energy Phys. 06 (2015) 170.

[29] E. Izaguirre and I. Yavin, Phys. Rev. D 92, 035014 (2015).

[30] L. Delle Rose, S. Khalil, and S. Moretti, Phys. Rev. D 96, 115024 (2017).

[31] M. D. Campos, D. Cogollo, M. Lindner, T. Melo, F. S. Queiroz, and W. Rodejohann, J. High Energy Phys. 08 (2017) 092.

[32] M. Abdullah, J. B. Dent, B. Dutta, G. L. Kane, S. Liao, and L. E. Strigari, Phys. Rev. D 98, 015005 (2018).

[33] E. Bertuzzo, S. Jana, P. A. N. Machado, and R. Zukanovich Funchal, Phys. Rev. Lett. 121, 241801 (2018).

[34] P. Souder and K. D. Paschke, Front. Phys. (Beijing) 11, 111301 (2016).

[35] M. S. Safronova, D. Budker, D. DeMille, D. F. J. Kimball, A. Derevianko, and C. W. Clark, Rev. Mod. Phys. 90, 025008 (2018).

[36] P. J. Fox, J. Liu, D. Tucker-Smith, and N. Weiner, Phys. Rev. D 84, 115006 (2011).

[37] G. C. Branco, P. M. Ferreira, L. Lavoura, M. N. Rebelo, M. Sher, and J. P. Silva, Phys. Rep. 516, 1 (2012).

[38] M. Deniz et al. (TEXONO Collaboration), Phys. Rev. D 81, 072001 (2010).

[39] H. B. Li et al. (TEXONO Collaboration), Phys. Rev. Lett. 90, 131802 (2003).

[40] H. T. Wong et al. (TEXONO Collaboration), Phys. Rev. D 75, 012001 (2007).

[41] J.-W. Chen, H.-C. Chi, H.-B. Li, C. P. Liu, L. Singh, H. T. Wong, C.-L. Wu, and C.-P. Wu, Phys. Rev. D 90, 011301 (2014).

[42] L. B. Auerbach et al. (LSND Collaboration), Phys. Rev. D 63, 112001 (2001).

[43] G. Bellini et al., Phys. Rev. Lett. 107, 141302 (2011).

[44] A. G. Beda, E. V. Demidova, A. S. Starostin, V. B. Brudanin, V. G. Egorov, D. V. Medvedev, M. V. Shirchenko, and T. Vylov, Phys. Part. Nucl. Lett. 7, 406 (2010).

[45] P. Vilain et al. (CHARM-II Collaboration), Phys. Lett. B 302, 351 (1993).

[46] S. Bilmis, I. Turan, T. M. Aliev, M. Deniz, L. Singh, and H. T. Wong, Phys. Rev. D 92, 033009 (2015).

[47] J. Benesch et al. (MOLLER Collaboration), arXiv:1411 .4088.

[48] T. Aoki et al., Appl. Phys. 123, 120 (2017).

[49] S. G. Porsev, K. Beloy, and A. Derevianko, Phys. Rev. Lett. 102, 181601 (2009).
[50] C. Bouchiat and P. Fayet, Phys. Lett. B 608, 87 (2005).

[51] G. Aad et al. (ATLAS Collaboration), Phys. Rev. D 92, 092001 (2015).

[52] M. Aaboud et al. (ATLAS Collaboration), J. High Energy Phys. 06 (2018) 166.

[53] T. Inami and C. S. Lim, Prog. Theor. Phys. 65, 297 (1981); 65, 1772(E) (1981).

[54] X.-G. He, J. Tandean, and G. Valencia, Eur. Phys. J. C 64, 681 (2009).

[55] J. Grygier et al. (Belle Collaboration), Phys. Rev. D 96, 091101 (2017).

[56] B. Aubert et al. (BABAR Collaboration), Phys. Rev. Lett. 102, 091803 (2009).

[57] R. Aaij et al. (LHCb Collaboration), Phys. Rev. D 95, 071101 (2017).

[58] A. V. Artamonov et al. (E949 Collaboration), Phys. Rev. Lett. 101, 191802 (2008).

[59] A. Alavi-Harati et al. (KTeV Collaboration), Phys. Rev. Lett. 93, 021805 (2004).

[60] A. Crivellin, G. D’Ambrosio, T. Kitahara, and U. Nierste, Phys. Rev. D 96, 015023 (2017).

[61] F. Bergsma et al. (CHARM Collaboration), Phys. Lett. B 157B, 458 (1985).

[62] M. J. Dolan, F. Kahlhoefer, C. McCabe, and K. Schmidt-Hoberg, J. High Energy Phys. 03 (2015) 171; 07 (2015) 103(E).

[63] M. W. Winkler, Phys. Rev. D 99, 015018 (2019).

[64] R. Essig, R. Harnik, J. Kaplan, and N. Toro, Phys. Rev. D 82, 113008 (2010).

[65] B. Dbrich, F. Ertas, F. Kahlhoefer, and T. Spadaro, Phys. Lett. B 790, 537 (2019).

[66] F. Bergsma et al. (CHARM Collaboration), Phys. Lett. B 128B, 361 (1983).

[67] M. Freytsis, Z. Ligeti, and J. Thaler, Phys. Rev. D 81, 034001 (2010).

[68] M. Tanabashi et al. (Particle Data Group), Phys. Rev. D 98, 030001 (2018).

[69] V. Khachatryan et al. (CMS Collaboration), J. High Energy Phys. 11 (2015) 018.

[70] V. Khachatryan et al. (CMS Collaboration), J. High Energy Phys. 12 (2015) 178.

[71] A. M. Sirunyan et al. (CMS Collaboration), Phys. Rev. Lett. 119, 141802 (2017).

[72] G. Aad et al. (ATLAS Collaboration), J. High Energy Phys. 03 (2016) 127.

[73] G. Aad et al. (ATLAS Collaboration), Phys. Rev. Lett. 114, 231801 (2015).

[74] M. Aaboud et al. (ATLAS Collaboration), Phys. Lett. B 759, 555 (2016).

[75] G. D'Ambrosio, G. Ecker, G. Isidori, and J. Portoles, J. High Energy Phys. 08 (1998) 004. 